\title{
Mortalidad y reclutamiento de árboles en un bosque secundario tardío del valle de Chanchamayo, Perú
}

\author{
Tree mortality and recruitment in a late secondary forest of the Chanchamayo \\ valley, Peru
}

José Giacomotti Tuezta ${ }^{1 \star \star}$ y Carlos Reynel Rodríguez ${ }^{1}$

\begin{abstract}
Resumen
El presente estudio evaluó los parámetros de mortalidad y reclutamiento de la dinámica forestal en una parcela permanente de una hectárea ubicada en un área de bosque secundario tardío a $1158 \mathrm{msnm}$ en el estrato premontano del valle de Chanchamayo, departamento de Junín, en la selva central del Perú. Fueron registrados todos los individuos con un diámetro a la altura de pecho (DAP) mayor o igual a $10 \mathrm{~cm}$, después de un periodo intercensal de 3.7 años. El establecimiento y primera medición de la parcela se efectuó en el año 2010, registrándose una población de 512 individuos. La remedición se realizó en el año 2014, encontrándose 35 individuos muertos y 59 reclutas, teniendo como población final a 536 individuos. Las especies que presentaron más individuos muertos fueron Trophis caucana (Pittier) C.C. Berg (19 individuos), Coussapoa villosa Poepp. \& Endl. (5 individuos), Cupania cinerea Poepp. (3 individuos), Cecropia membranacea Trécul (2 individuos) y Pseudobombax munguba (Mart.) Dugand (2 individuos). Las especies que presentaron el mayor número de individuos reclutas fueron Trophis caucana (30 reclutas), Guarea guidonia (L.) Sleumer (5 reclutas), Bunchosia armeniaca (Cav.) DC. (3 reclutas) y Trema micrantha (L.) Blume (3 reclutas). Se registró una tasa anual de mortalidad de $1.91 \%$ y una tasa anual de reclutamiento de $3.15 \%$. La vida media y el tiempo de duplicación para este bosque se calcularon en 36 años y en 22 años respectivamente. En base a los resultados se concluye que el bosque presenta una dinámica moderadamente alta y está incrementando su densidad arbórea.
\end{abstract}

Palabras clave: Dinámica forestal, tasa de mortalidad, tasa de reclutamiento.

\footnotetext{
${ }^{1}$ Herbario Forestal MOL, Facultad de Ciencias Forestales, Universidad Nacional Agraria La Molina, Av. La Molina s/n, La Molina, Lima, Perú.

* Autor de Correspondencia: josegiacomottituezta@gmail.com
} 


\begin{abstract}
The present study evaluated the parameters of mortality and recruitment of the forest dynamics at a permanent plot of one hectare located in a late secondary forest area, at 1158 $\mathrm{m}$ above the sea level (masl) in the premontane stratum of the Chanchamayo Valley, Department of Junín, in the central jungle of Peru. All individuals with a diameter at breast height $(\mathrm{DBH})$ greater than or equal to $10 \mathrm{~cm}$ were recorded, after an intercensal period of 3.7 years. The establishment and the first measurement of the plot was performed in 2010, registering a population of 512 individuals. The remeasurement was carried out in 2014, finding 35 dead individuals and 59 recruits, having as final population 536 individuals. The species that presented more dead individuals were Trophis caucana (Pittier) C.C. Berg (19 individuals), Coussapoa villosa Poepp. \& Endl. (5 individuals), Cupania cinerea Poepp. (3 individuals), Cecropia membranacea Trécul (2 individuals) and Pseudobombax munguba (Mart.) Dugand (2 individuals). The species that presented the highest number of recruits were Trophis caucana (30 recruits), Guarea guidonia (L.) Sleumer (5 recruits), Bunchosia armeniaca (Cav.) DC. (3 recruits) and Trema micrantha (L.) Blume (3 recruits). Recording the annual mortality rate of $1.91 \%$ and the annual recruitment rate of $3.15 \%$. The half-life and duplication time for this forest were calculated at 36 years and 22 years respectively. Based on the results, the conclusion is that the forest presents a moderately high dynamics and is increasing its arboreal density.
\end{abstract}

Key words: Forest dynamics, mortality rate, recruitment rate.

\section{Introducción}

En los bosques tropicales ocurren cambios en el tiempo que modifican su composición, estructura y diversidad, estos se pueden documentar a través de mediciones sucesivas en parcelas permanentes (Ramírez et al. 2002). Las parcelas permanentes de monitoreo de vegetación sirven como una metodología de investigación para estudiar la dinámica forestal y la regeneración de los bosques. En estas se realizan remediciones cada cierto tiempo para documentar la mortalidad y reclutamiento de individuos, los ritmos de crecimiento en el diámetro de los árboles y el ingreso de especies que no habían estado presentes en el bosque (Aguilar y Reynel 2009).

El reclutamiento en un bosque está representado por el número de individuos que alcanzan el diámetro mínimo de registro a lo largo de un periodo intercensal. Además, se considera como una manifestación de la fecundidad de las especies, al expresar el crecimiento y sobrevivencia de los juveniles de una población forestal (Swaine et al. 1987). Por otro lado, la mortalidad se puede expresar como la cantidad o porcentaje de individuos que mueren después de un periodo de tiempo determinado. Ambos procesos son fundamentales para mantener la regeneración natural como la diversidad vegetal, y de la relación entre los dos depende la densidad de árboles del bosque (Quinto et al. 2009).

Los bosques secundarios en los trópicos húmedos se caracterizan porque se forman debido a disturbios o perturbaciones a sus ecosistemas causados de forma natural (por fenómenos atmosféricos, geológicos, por la fauna silvestre, etc.) o por acción del hombre como actor principal (origen antrópico). Los bosques secundarios originados por actividades humanas son más comunes y ocupan hoy en día, una mayor superficie que los ocasionados por perturbaciones naturales, además de tener implicancias mayores sobre el uso de tierra, el desarrollo rural y la conservación de recursos naturales en general (Smith et al. 1997).

El presente estudio está centrado en la remedición en una parcela permanente de una hectárea en el valle de Chanchamayo, en el De- 
partamento de Junín, en el estrato premontano entre los 800 y $1500 \mathrm{msnm}$. La parcela fue establecida en el año 2010 y después de un periodo intercensal de 3.7 años, se remidió en el año 2014. En este contexto, el propósito de este estudio fue evaluar la mortalidad y el reclutamiento de los árboles en un área de bosque húmedo secundario tardío y determinar la tasa anual de mortalidad, la tasa anual de reclutamiento, la vida media del bosque y su tiempo de duplicación.

\section{Materiales y Métodos}

El estudio se realizó en una parcela de investigación al interior del Instituto Regional de Desarrollo Fundo La Génova de la Universidad Nacional Agraria La Molina (UNALM), ubicada en un área de bosque secundario tardío, a una altitud de $1158 \mathrm{msnm}$ en el estrato premontano. Políticamente se localiza en el distrito de San Ramón, provincia de Chanchamayo, departamento de Junín. Según el Mapa Ecológico del Perú elaborado por la ONERN
(1976), basado en las zonas de vida desarrolladas por Holdridge (1978), el área de estudio se encuentra en la zona de vida bosque húmedo premontano Tropical (bh-PT). La parcela tiene una extensión de 1 ha $(100 \mathrm{~m}$ x $100 \mathrm{~m})$ y está dividida en 25 subparcelas de $20 \mathrm{~m}$ x $20 \mathrm{~m}$. Su vértice noroeste tiene las siguientes coordenadas UTM: 460947 m E y 8772688 m N (ver Figura 1).

Para realizar la remedición de la parcela se empleó la metodología propuesta por la Red Amazónica de Inventarios Forestales (RAINFOR) (Phillips y Baker 2006). Los árboles fueron clasificados como muertos, reclutas y sobrevivientes. En cuanto al tipo de mortalidad, los árboles muertos se tipificaron en "muerto caído", "muerto parado", "muerto roto" y "presumido muerto" (Phillips y Baker 2006). Los reclutas son todos los nuevos individuos de la parcela que alcanzaron un DAP igual o mayor a los $10 \mathrm{~cm}$, a lo largo del periodo intercensal. Los sobrevivientes son aquellos que fueron inventariados en el primer censo (año 2010) y registrados en la segunda medición

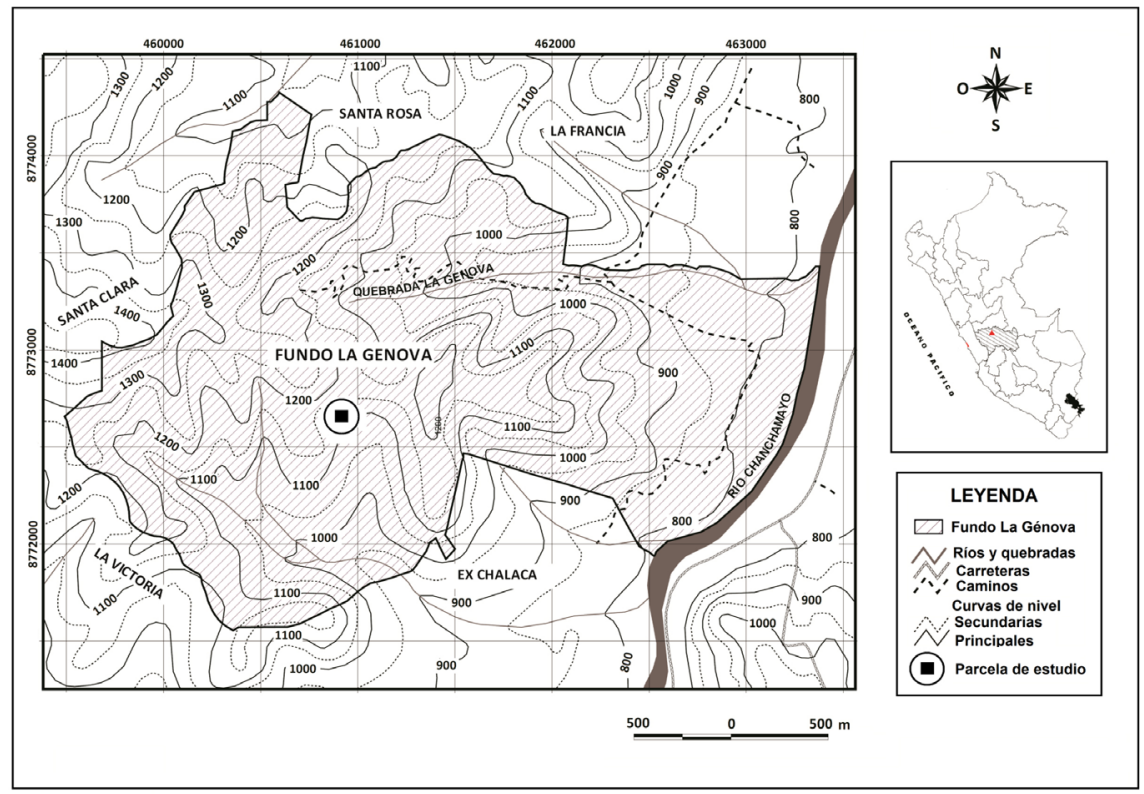

Figura 1. Ubicación de la Parcela Permanente de estudio en el Fundo La Génova, Chanchamayo, Junín. Modificado a partir de Palacios y Reynel (2011). 


\begin{tabular}{|l|l|}
\hline Cálculo de la tasa anual de mortalidad & Cálculo de la tasa anual de reclutamiento \\
\hline Fórmula: & Fórmula: \\
$\left.\qquad \frac{\ln \left(\frac{N_{O}}{N_{S}}\right)}{t}\right]$ & $\mathrm{r}=\left[\frac{\ln \left(\frac{N_{f}}{N_{S}}\right)}{t}\right]$ \\
\hline $\begin{array}{l}\text { Donde: } \\
\mathrm{m}=\text { Tasa anual de mortalidad en \% } \\
\mathrm{N}_{\mathrm{o}}=\text { Número de individuos inicialmente } \\
\text { inventariados } \\
\mathrm{N}_{\mathrm{s}}=\text { Número de individuos sobrevivientes } \\
\mathrm{t}=\text { Intervalo "t" de tiempo } \\
\ln =\text { Logaritmo neperiano }\end{array}$ & $\begin{array}{l}\text { Donde: } \\
\mathrm{N}=\text { Tasa anual de reclutamiento en } \% \\
\text { del inventario } \\
\mathrm{N}_{\mathrm{s}}=\text { Número de individuos sobrevivientes } \\
\mathrm{t}=\text { Intervalo "t" de tiempo } \\
\text { ln= Logaritmo neperiano }\end{array}$ \\
\hline
\end{tabular}

Cuadro 1. Cálculo de las tasas de mortalidad y reclutamiento.

\begin{tabular}{|l|l|}
\hline \multicolumn{1}{|c|}{ Cálculo de la vida media } & Cálculo del tiempo de duplicación \\
\hline Fórmula: & Fórmula: \\
$\qquad \begin{array}{l}t_{0.5}=\frac{\ln 0.5}{\ln (1-m)} \\
\quad t_{2}=\frac{\ln 2}{\ln (1+r)}\end{array}$ \\
\hline $\begin{array}{l}\text { Donde: } \\
t_{0.5}=\text { Vida media en años } \\
\ln =\text { Logaritmo neperiano } \\
\mathrm{m}=\text { Tasa anual de mortalidad }\end{array}$ & $\begin{array}{l}\text { Donde: } \\
\mathrm{t}_{2}=\text { Tiempo de duplicación en años } \\
\ln =\text { Logaritmo neperiano } \\
\mathrm{r}=\text { Tasa anual de reclutamiento }\end{array}$ \\
\hline
\end{tabular}

Cuadro 2. Cálculo de la vida media y del tiempo de duplicación.

(año 2014) luego de un periodo intercensal de 3.7 años. Todos los individuos a los cuales no se les pudieron realizar plenamente una identificación botánica en el campo, fueron colectados y sus muestras botánicas fueron identificadas en el Herbario Forestal MOL de la Facultad de Ciencias Forestales de la UNALM.

Se procesó la información recogida en el campo mediante una base de datos utilizando el programa de computación Excel, para poder clasificar a los individuos según su condición de recluta, muerto o sobreviviente. Las tasas anuales de mortalidad y reclutamiento, así como la vida media del bosque y el tiempo de duplicación, se calcularon usando las fórmulas matemáticas mostradas en los Cuadros 1 y 2 (Swaine y Lieberman 1987; Phillips et al. 1994; Londoño y Jiménez 1999; Nebel et al. 2000).

\section{Resultados}

En el año 2010 la primera medición de la parcela dio como resultado un total de 512 individuos. Después de un periodo intercensal de 3.7 años, la población del bosque aumentó a 536 individuos. En ambos censos la especie forestal más abundante en la parcela fue Trophis caucana (Pittier) C.C. Berg con 198 individuos en el primer censo y 209 individuos en el segundo censo.

\section{Mortalidad}

En la remedición del año 2014 se registraron 35 individuos muertos agrupados en 9 especies y 8 familias. Los valores de mortalidad en cada subparcela van desde 0 hasta 5 individuos muertos, con un promedio de 1.4 ár- 
boles muertos por subparcela. En 19 de las 25 subparcelas se registró al menos un individuo muerto (Cuadro 3). La especie que presenta mayor mortalidad es Trophis caucana con el 54\% (19 individuos), seguida de Coussapoa villosa Poepp. \& Endl. con el 14\% (5 individuos), el resto de especies se detalla en el Cuadro 4. En cuanto al tipo de mortalidad, el $63 \%$ de los árboles muertos encontrados se ubican dentro de la categoría de "muerto caído", como se aprecia en el Cuadro 5.

\section{Reclutamiento}

Se registraron en total 59 individuos reclutas agrupados en 18 especies y 13 familias. Los valores de reclutamiento en cada subparcela varían de 0 a 5 reclutas, haciendo un promedio de 2.36 reclutas por subparcela. Se reportó al menos un individuo recluta en 20 de las 25 subparcelas (Cuadro 3). La especie que presenta el mayor reclutamiento es Trophis caucana con el 51\% (30 reclutas), seguida de Guarea guidonia (L.) Sleumer con 8\% (5 reclutas), el resto de especies se detalla en el Cuadro 6.

\section{Tasa anual de mortalidad y de reclutamiento}

La parcela de estudio registró una tasa anual de mortalidad de $1.91 \%$ y una tasa anual de reclutamiento de $3.15 \%$, después de un período intercensal de 3.7 años (Cuadro 3). En base a la tasa anual de mortalidad, se calculó la vida media del bosque en 36 años. A partir de la tasa anual de reclutamiento, el tiempo de duplicación del bosque se calculó en 22 años.

\section{Discusión}

Luego del periodo intercensal de 3.7 años, se registró la muerte de 35 individuos de un total de 512 árboles. La especie que presentó la mayor mortalidad fue Trophis caucana con 19 individuos muertos, especie reportada previamente en bosques secundarios de la zona de estudio (Echia 2013). La mayor cantidad de árboles muertos correspondieron a la categoría de "muerto caído" con un 63\% (22 individuos). Resultado que se asemeja al encontrado por Aguilar (2009) en un bosque primario a 2100 msnm del valle de Chanchamayo, donde el
50\% de árboles muertos (13 individuos), se reportan dentro de este tipo de muerte. Buttgenbach et al. (2013) señalan que esta condición de "muerto caído" está asociada a factores exógenos de carácter físico como vientos fuertes, topografía y suelos inestables. Esto sugiere que estos factores favorecen el desprendimiento de los árboles del suelo y su consecuente caída, determinando la mortalidad. La categoría "muerto parado" abarca al 28\% de los árboles muertos (10 individuos). Buttgenbach et al. (2013) indican que este tipo de mortalidad está ligado a factores endógenos (fitosanitarios, competencia), asociados especialmente a causas biológicas. Lugo y Scatena (1996) señalan que una de las causas de la mortalidad de los árboles se presenta por acción de sustancias tóxicas, agentes patógenos y parásitos. Lo que sugiere que estos factores endógenos influyen moderadamente en la muerte de los árboles en el bosque estudiado.

La tasa anual de mortalidad de $1.91 \%$ en el área estudiada, indica que el bosque presenta una "mortalidad de trasfondo" ( $<5 \%$ año$\left.{ }^{-1}\right)$. Esta se caracteriza por no registrar fuertes perturbaciones, a diferencia de la "mortalidad catastrófica" ( $>5 \%$ año $\left.^{-1}\right)$, producto de fuertes perturbaciones en el bosque (Lugo y Scatena 1996). La "mortalidad de trasfondo" contiene eventos a pequeña escala que ocasionan cambios graduales en el bosque (Lugo y Scatena 1996; Londoño y Jiménez 1999; Quinto et al. 2009). Además, puede estar influenciada por la posición, edad y tamaño de los árboles en el dosel, y por la etapa sucesional del bosque (Palacios 2017). Esto sugiere que en este bosque no sucedieron cambios significativos que influyeron en la mortalidad.

En 27 parcelas amazónicas de RAINFOR en bosques tropicales de Brasil, Ecuador y Venezuela se reportan tasas anuales de mortalidad que van desde $0.37 \%$ hasta $3.25 \%$, estos valores han sido registrados en dos intervalos de medición y en periodos intercensales que van en promedio de 4 a 13 años (Lewis et al. 2004). Para el Perú, RAINFOR reporta en 23 parcelas amazónicas de bosques tropicales, tasas anuales de mortalidad que van de $1.03 \%$ a $3.97 \%$, 


\begin{tabular}{|c|c|c|c|c|c|c|c|}
\hline $\begin{array}{c}\text { Sub } \\
\text { parcela }\end{array}$ & $\begin{array}{l}\text { Población } \\
\text { Inicial } \\
(2010)(\mathrm{No})\end{array}$ & $\begin{array}{l}\text { Muertos } \\
\text { (Nm) }\end{array}$ & $\begin{array}{c}\text { Sobrevivientes } \\
\text { (Ns) }\end{array}$ & $\begin{array}{l}\text { Reclutas } \\
\text { (Nr) }\end{array}$ & $\begin{array}{c}\text { Población } \\
\text { Final } \\
(2014)(\mathrm{Nf})\end{array}$ & $\begin{array}{c}\text { Tasa de } \\
\text { mortalidad } \\
\text { anual \% } \\
\text { (m) }\end{array}$ & $\begin{array}{c}\text { Tasa de } \\
\text { reclutamiento } \\
\text { anual } \%(r)\end{array}$ \\
\hline 1 & 37 & 1 & 36 & 3 & 39 & 0.74 & 2.16 \\
\hline 2 & 22 & 0 & 22 & 3 & 25 & 0.00 & 3.45 \\
\hline 3 & 32 & 1 & 31 & 5 & 36 & 0.86 & 4.04 \\
\hline 4 & 22 & 0 & 22 & 4 & 26 & 0.00 & 4.51 \\
\hline 5 & 14 & 1 & 13 & 2 & 15 & 2.00 & 3.87 \\
\hline 6 & 16 & 1 & 15 & 2 & 17 & 1.74 & 3.38 \\
\hline 7 & 8 & 0 & 8 & 1 & 9 & 0.00 & 3.18 \\
\hline 8 & 16 & 1 & 15 & 1 & 16 & 1.74 & 1.74 \\
\hline 9 & 18 & 3 & 15 & 0 & 15 & 4.93 & 0.00 \\
\hline 10 & 15 & 1 & 14 & 0 & 14 & 1.86 & 0.00 \\
\hline 11 & 31 & 1 & 30 & 0 & 30 & 0.89 & 0.00 \\
\hline 12 & 22 & 2 & 20 & 1 & 21 & 2.58 & 1.32 \\
\hline 13 & 24 & 2 & 22 & 3 & 25 & 2.35 & 3.45 \\
\hline 14 & 31 & 4 & 27 & 3 & 30 & 3.73 & 2.85 \\
\hline 15 & 13 & 5 & 8 & 2 & 10 & 13.12 & 6.03 \\
\hline 16 & 17 & 2 & 15 & 4 & 19 & 3.38 & 6.39 \\
\hline 17 & 21 & 1 & 20 & 3 & 23 & 1.32 & 3.78 \\
\hline 18 & 22 & 1 & 21 & 5 & 26 & 1.26 & 5.77 \\
\hline 19 & 15 & 3 & 12 & 3 & 15 & 6.03 & 6.03 \\
\hline 20 & 28 & 2 & 26 & 3 & 29 & 2.00 & 2.95 \\
\hline 21 & 19 & 2 & 17 & 0 & 17 & 3.01 & 0.00 \\
\hline 22 & 25 & 0 & 25 & 5 & 30 & 0.00 & 4.93 \\
\hline 23 & 5 & 0 & 5 & 5 & 10 & 0.00 & 18.73 \\
\hline 24 & 20 & 1 & 19 & 0 & 19 & 1.39 & 0.00 \\
\hline 25 & 19 & 0 & 19 & 1 & 20 & 0.00 & 1.39 \\
\hline $\begin{array}{c}\text { Total } \\
\text { Parcela }\end{array}$ & 512 & 35 & 477 & 59 & 536 & 1.91 & 3.15 \\
\hline
\end{tabular}

Cuadro 3. Mortalidad y reclutamiento de individuos por subparcelas.

registradas en dos intervalos de medición y en periodos intercensales que van en promedio de 4 a 15 años (Lewis et al. 2004). En la llanura amazónica de Ucayali (Perú) se estudiaron tres parcelas en 4 años, registrando en promedio tasas anuales de mortalidad que van de $2.20 \%$ hasta 3.16\% (Nebel et al. 2000). En el valle de Chanchamayo, en selva central del Perú, Aguilar (2008) en un bosque montano reporta una tasa anual de mortalidad de $1.07 \%$ (3.58 años de periodo intercensal), y Buttgenbach et al. (2013) en un bosque premontano, reportan 
Mortalidad y reclutamiento de árboles de un bosque secundario tardío del valle de Chanchamayo, Perú

\begin{tabular}{|c|l|c|c|}
\hline $\mathbf{N}^{\mathbf{0}}$ & \multicolumn{1}{|c|}{ Especie muerta } & $\begin{array}{c}\text { Número de } \\
\text { Individuos }\end{array}$ & Porcentaje (\%) \\
\hline 1 & Trophis caucana (Pittier) C.C. Berg & 19 & 54 \\
\hline 2 & Coussapoa villosa Poepp. \& Endl. & 5 & 14 \\
\hline 3 & Cupania cinerea Poepp. & 3 & 9 \\
\hline 4 & Cecropia membranacea Trécul & 2 & 6 \\
\hline 5 & Pseudobombax munguba (Mart.) Dugand & 2 & 6 \\
\hline 6 & Chimarrhis hookeri K. Schum. & 1 & 3 \\
\hline 7 & Cyathea sp. & 1 & 3 \\
\hline 8 & Mauria heterophylla Kunth & 1 & 3 \\
\hline 9 & Sapium glandulosum (L.) Morong & 1 & $\mathbf{1 0 0}$ \\
\hline & Total & $\mathbf{3 5}$ & \\
\hline
\end{tabular}

Cuadro 4. Mortalidad por especie en la parcela.

\begin{tabular}{|c|c|c|}
\hline Tipo de mortalidad & $\begin{array}{c}\text { Número de individuos } \\
\text { muertos }\end{array}$ & Porcentaje (\%) \\
\hline Muerto caído & 22 & 63 \\
\hline Muerto parado & 10 & 28 \\
\hline Muerto roto & 2 & 6 \\
\hline Presumido muerto & 1 & 3 \\
\hline Total & $\mathbf{3 5}$ & $\mathbf{1 0 0}$ \\
\hline
\end{tabular}

Cuadro 5. Tipos de mortalidad en la parcela.

una tasa anual de anual de mortalidad de $2.16 \%$ (6 años de periodo intercensal). La tasa anual de mortalidad de la parcela de estudio, $1.91 \%$, se encuentra dentro del rango medio de estos estudios, con lo cual, no hay una disminución importante a nivel de la población del bosque.

En este estudio se registraron 59 reclutas con un DAP mínimo de $10 \mathrm{~cm}$. Las especies que presentan mayor reclutamiento como Trophis caucana (30 reclutas), Guarea guidonia (5 reclutas), Bunchosia armeniaca (Cav.) DC. (3 reclutas) y Trema micrantha (L.) Blume (3 reclutas), han sido reportadas en bosques secundarios de la zona de estudio (Reynel y León 1989; Echia 2013). Trophis caucana y Trema micrantha también son reportadas por Buttgenbach et al. (2013) como las especies que presentaron mayor reclutamiento en un bosque premontano de Chanchamayo en la selva central del Perú.

La tasa anual de reclutamiento del bosque estudiado es de $3.15 \%$. RAINFOR reporta en bosques tropicales de Brasil, Ecuador y Venezuela tasas anuales de reclutamiento entre $0.35 \%$ a $4.64 \%$, registradas en dos intervalos de medición y en periodos intercensales que van en promedio de 4 a 13 años (Lewis et al. 2004). En bosques tropicales del Perú, RAINFOR reporta tasas anuales de reclutamiento que van de $1.36 \%$ a $3.90 \%$, registradas en dos intervalos de medición y en periodos intercensales que van 


\begin{tabular}{|c|c|c|c|}
\hline $\mathbf{N}^{\mathbf{o}}$ & Especie recluta & $\begin{array}{l}\text { Número de Indi- } \\
\text { viduos }\end{array}$ & Porcentaje (\%) \\
\hline 1 & Trophis caucana (Pittier) C.C. Berg & 30 & 51 \\
\hline 2 & Guarea guidonia (L.) Sleumer & 5 & 8 \\
\hline 3 & Bunchosia armeniaca (Cav.) DC. & 3 & 5 \\
\hline 4 & Trema micrantha (L.) Blume & 3 & 5 \\
\hline 5 & Calliandra arborea Standl. & 2 & 3 \\
\hline 6 & Cecropia sp. & 2 & 3 \\
\hline 7 & Coussapoa villosa Poepp. \& Endl. & 2 & 3 \\
\hline 8 & Heliocarpus americanus L. & 2 & 3 \\
\hline 9 & Chimarrhis hookeri $\mathrm{K}$. Schum. & 1 & 2 \\
\hline 10 & Cupania cinerea Poepp. & 1 & 2 \\
\hline 11 & Erythrina ulei Harms & 1 & 2 \\
\hline 12 & Guazuma ulmifolia Lam. & 1 & 2 \\
\hline 13 & Macrocnemum roseum (Ruiz \& Pav.) Wedd. & 1 & 2 \\
\hline 14 & Mauria heterophylla Kunth & 1 & 2 \\
\hline 15 & Miconia calvescens DC. & 1 & 2 \\
\hline 16 & Prunus debilis Koehne & 1 & 2 \\
\hline 17 & Senegalia polyphylla (DC.) Britton & 1 & 2 \\
\hline \multirow[t]{2}{*}{18} & Triplaris setosa Rusby & 1 & 2 \\
\hline & Total & 59 & 100 \\
\hline
\end{tabular}

Cuadro 6. Reclutamiento por especie en la parcela.

en promedio de 4 a 15 años (Lewis et al. 2004). En la llanura amazónica de Ucayali (Perú) se estudiaron 3 parcelas en 4 años, que registraron en promedio tasas anuales de reclutamiento de $2.99 \%$ a $4.57 \%$ (Nebel et al. 2000). Estudios en la selva central del Perú en el valle de Chanchamayo, reportan tasas anuales de reclutamiento de $2.94 \%$ en un bosque montano en un periodo intercensal de 3.58 años (Aguilar 2008) y de $3.27 \%$ en un bosque premontano en un periodo intercensal de 6 años (Buttgenbach et al. 2013). La tasa de reclutamiento de la parcela de estudio se encuentra dentro de los rangos superiores reportados en los bosques mencionados. Además, es similar a los valores registrados en la selva central del Perú. El reclutamiento de árboles muestra la capacidad de un bosque de recuperarse rápidamente manteniendo la estabilidad ecológica de los ecosistemas (Quinto et al. 2009). Este resultado sugiere que el bosque estudiado muestra un incremento en la densidad de su población.

Aguilar (2008) y Buttgenbach et al. (2013) en la selva central del Perú, reportan tasas de reclutamiento superiores a las tasas de mortalidad. En un bosque pluvial tropical de Colombia, Quinto et al. (2009), sugieren un probable equilibrio dinámico en el bosque, cuando reportan un balance entre la tasa anual de mortalidad (1.39\%) y la tasa anual de reclutamiento $(1.2 \%)$. Nebel et al. (2000) señalan que los 
bosques están en crecimiento cuando las tasas anuales de reclutamiento son superiores a tasas anuales de mortalidad. En este estudio, la tasa de reclutamiento es mayor que la tasa de mortalidad (3.15\% y $1.91 \%$ respectivamente), lo que podría evidenciar que el bosque ostenta una buena capacidad de regeneración natural.

La vida media es el tiempo que requiere el bosque para que su población se reduzca a la mitad, en presente estudio ha sido calculada en 36 años. El tiempo de duplicación es aquel que necesita la población del mismo bosque para duplicarse, el cual se calculó en 22 años, lo que expresa que el bosque necesita relativamente pocos años para aumentar su población. Según Korning y Balslev (1994), un bosque en equilibrio es aquel en donde la vida media y el tiempo de duplicación serían iguales. Los resultados obtenidos sugieren que el bosque en estudio todavía no alcanza el equilibrio, debido a que su densidad arbórea aumenta más rápido de lo que disminuye su población.

\section{Conclusiones}

El bosque presenta una dinámica moderadamente alta, con una tasa de reclutamiento mayor a la tasa anual de mortalidad, por lo que aún no alcanza el equilibrio dinámico y muestra una tendencia de incremento en su densidad arbórea.

El bosque presenta niveles de mortalidad que no ocasionan una disminución significativa de su población. Por lo tanto, la abundancia (número de individuos) se ha incrementado de forma moderada en el periodo intercensal de 3.7 años, demostrando que el bosque evaluado presenta una buena capacidad en la recuperación de su población.

\section{Agradecimientos}

Los autores agradecen de forma especial a Aniceto Daza Yomona por su trabajo brindado en el establecimiento y remedición de la parcela de investigación. A los trabajadores del Fundo La Génova en Chanchamayo y al Laboratorio de Dendrología y Herbario de la Facultad de
Ciencias Forestales de la Universidad Nacional Agraria La Molina. Asimismo, agradecemos a Sonia Palacios Ramos, Rocío Armey Malpartida, Italo Revilla Pantigoso y Akira Wong Sato. Esta investigación fue financiada por Cienciactiva, CONCYTEC.

\section{Bibliografía}

Aguilar, M. 2008. Evaluación de la dinámica forestal en el bosque montano de Pichita APRODES, en Chanchamayo, selva central del Perú. Tesis Mg. Sc. Lima, Perú, UNALM. 129 p.

Aguilar, M; Reynel, C. 2009. Dinámica Forestal y Regeneración en un bosque montano nublado de la Selva Central del Perú (localización Puyu Sacha, valle de Chanchamayo, Dp. Junín, 2100 msnm) Herbario de la Facultad de Ciencias Forestales de la Universidad Nacional Agraria La Molina. Lima, Perú. 167 p.

Buttgenbach, H; Vargas, C; Reynel, C. 2013. Dinámica forestal en un bosque premontano del valle de Chanchamayo (Dpto. de Junín, 1200 msnm). Herbario de la Facultad de Ciencias Forestales de la Universidad Nacional Agraria La Molina. Lima, Perú. 103 p.

Echia, E. 2013. Composición y diversidad de la flora leñosa en bosques secundarios generados a partir de quemas en el Valle de Chanchamayo, Junín. Tesis Ing. Forestal. Lima, Perú, UNALM. 133 p.

Holdridge, LR. 1978. Ecología basada en las zonas de vida. Instituto Interamericano de Cooperación para la Agricultura. San José, Costa Rica. 216 p.

Korning, J; Balslev, H. 1994. Growth rates and mortality patterns of tropical lowland tree species and the relation to forest structure in Amazonian Ecuador. Journal of Tropical Ecology 10:151-166.

Lewis, SL; Phillips, OL; Baker, TR; Lloyd, J; Mahli, Y; Almeida, S; Higuchi, N; Laurance, WF; Neill, DA; Silva, JNM; Terbogh, J; Torres Lezama, A; Vásquez Martínez, R; Brown, S; Chave, J; Kuebler, C; Nuñez Vargas, P; Vicenti, B. 2004. Concerted changes in tropical fo- 
rest structure and dynamics: evidence from 50 South American long-term plots. The Royal Society 359:421-436.

Londoño, C; Jiménez, E. 1999. Efecto del tiempo entre los censos sobre la estimación de las tasas anuales de mortalidad y de reclutamiento de árboles (periodos de 1, 4 y 5 años). Crónica Forestal y del Medio Ambiente 14(1):41-58.

Lugo, AE; Scatena, FN. 1996. Background and Catastrophic Tree Mortality in Tropical Moist, Wet, and Rain Forests. Biotropica 28(4a):585-599.

Nebel, G; Kvist, LP; Vanclay, JK; Vidaurre, H. 2000. Dinámica de los bosques de la llanura aluvial inundable de la Amazonía Peruana: Efectos de las perturbaciones e implicancias para su manejo y conservación. Folia Amazonica 11(1-2):65-97.

ONERN, 1976. Mapa ecológico del Perú. Guía explicativa Oficina Nacional de Evaluación de Recursos Naturales del Perú. Lima, Perú. 146 p.

Palacios, S. 2016. Dinámica forestal en una formación forestal subxerófila en el Valle de Chanchamayo, dpto. Junín - Perú. Tesis Mg. Sc. Lima, Perú, UNALM. 146 p.

Palacios, S; Reynel, C. 2011. Una formación vegetal subxerófila en el Valle de Chanchamayo, Dp. de Junín. CED-FDA, APRODES. Lima, Perú. 70 p.

Phillips, OL; Baker, TR. 2006. Manual de campo para la remedición y establecimiento de parcelas RAINFOR. ECI - Oxford University. $18 \mathrm{p}$.

Phillips, OL; Hall, P; Gentry, AH; Sawyer, SA; Vásquez, R. 1994. Dynamics and species richness of tropical rain forests. Proc. Nat. Acad. Sci. USA 91:2805-2809.

Ramírez, H; Torres, A; Serrano, J. 2002. Mortalidad y reclutamiento de árboles en un bosque nublado de la Cordillera de Los Andes, Venezuela. ECOTROPICOS 15(2): 177-184.

Reynel, C; León, J. 1989. Especies forestales de los bosques secundarios de Chanchamayo (Perú). UNALM/UT/CIID. Lima, Perú. 173 p.
Quinto, H; Rengifo, R; Ramos, Y. 2009. Mortalidad y reclutamiento de árboles en un bosque pluvial tropical de Chocó (Colombia). Revista Facultad Nacional de Agronomía - Medellín (62)1:4855-4868.

Smith, J; Sabogal, C; de Jong, W; Kaimowitz, D. 1997. Bosques secundarios como recurso para el desarrollo rural y la conservación ambiental en los trópicos de América Latina. Center for International Forestry Research CIFOR. Bogor, Indonesia. 38 p.

Swaine, MD; Lieberman, D. 1987. Note on the calculation of mortality rates. Journal of Tropical Ecology 3(Special suplement): ii-iii.

Swaine, MD; Lieberman, D; Putz, FE. 1987. The dynamics of tree populations in tropical forest: a review. Journal of Tropical Ecology 3: 359-366.

Presentado: 09/05/2018 Aceptado: 20/06/2018 